\title{
Collaboration in 3D Virtual Worlds: a protocol for case study research
}

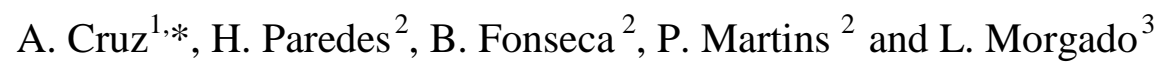 \\ ${ }^{1}$ INESC TEC and Instituto Politécnico de Viseu, Portugal \\ ${ }^{2}$ INESC TEC and UTAD, Vila Real, Portugal, \\ ${ }^{3}$ INESC TEC and Universidade Aberta, Coimbra, Portugal
}

\begin{abstract}
Three-dimensional virtual worlds have been growing fast in number of users, and are used for the most diverse purposes. In collaboration, they are used with good results due to features such as immersion, interaction capabilities, use of avatar embodiment, and physical space. In the particular cases of avatar embodiment and physical space, these features support nonverbal communication, but its impact on collaboration is not well known. In this work we present a protocol for case study research and its creation process, which aims to assert itself as a tool to collect data on how nonverbal communication influences collaboration in three-dimensional virtual worlds. We define the propositions and units of analysis, and a pilot case to validate them. Then, two cases are analysed under the created protocol. Most of the propositions found chains of evidences supporting them.
\end{abstract}

Keywords: virtual worlds, collaboration, immersion, interaction, communication, nonverbal, case study, pilot case.

Received on 29 October 2015, accepted on 8 January 2016, published on 27 June 2016

Copyright (9) 2016 A. Cruz et al., licensed to EAI. This is an open access article distributed under the terms of the Creative Commons Attribution licence (http://creativecommons.org/licenses/by/3.0/), which permits unlimited use, distribution and reproduction in any medium so long as the original work is properly cited.

doi: 10.4108/eai.27-6-2016.151521

\section{Introduction}

Three-dimensional virtual worlds (3DVW) have been used for collaboration in several areas such as education, training, and distance learning [1-5], decision making and planning [6-8], project management [3;7], and information systems [3;9]. 3DVW possess features that promote interaction and an immersive environment making them suitable for collaboration [3;9], with the most diverse objectives such as work, social interaction or gaming, are found in World of Warcraft and Second Life [10-13].

The immersive environment, as well as other features of 3DVW, is also responsible for a sensation felt by users, known as Presence, which occurs when they experience the virtual world without acknowledgment of the mediation of the technology [14]. It is believed that Presence improves collaboration [15], and Romano et al. [16] affirm that collaboration is related to a strong sense of presence shared by collaborators. In the knowledge

"Corresponding author. Email: cruz.armando1@ sapo.pt area that studies the phenomenon of Presence, it is recognized that immersion [16-21], nonverbal communication [22-26], and interaction [27-29] are important for Presence, with the potential for collaboration enhancement.

Nonverbal communication, including clues of presence related to social aspects, such as proximity, orientation of the avatar, focus, eye gaze, eye contact, physical appearance, and the use of avatar itself, strengthen the sense of presence and are important for collaboration [23]. Besides its importance for communication [23;25;26;29], nonverbal communication also improves awareness [23]. In the case of the field of vision, techniques of manipulation and navigation capabilities improve interaction, as well as the immersive environment created by $3 \mathrm{DVW}$, and facilitate cooperative tasks [19]. These facts clearly show a relationship between collaboration and Presence since communication, awareness, interaction and cooperation are directly related to collaboration. Nevertheless, there is a lack of literature on how nonverbal communication cues influence collaboration. 
The theoretical framework of the field of Computer Supported Cooperative Work (CSCW) provides a starting point for this work. However, CSCW taxonomies fail to properly address the key features of 3DVW, confounding them with other quite distinct systems [30]. In this paper, we present a research instrument that enables data collection on how nonverbal communication in 3DVW influences collaboration, thus contributing to improve the theoretical framework of CSCW in its ability to classify 3DVW. Generally, we intend to observe a user's behavior and the effect it appears to have on other users' behaviors. With this, we expect to relate some behaviors to specific effects, within a collaboration context, thus achieving data that may contribute to a better understanding of how nonverbal communication cues in 3DVW influence collaboration. The focus on case study research is due to the complex nature of the analysis of users' behaviors. This proposal establishes the first two steps of the case study methodology according to Yin [31], as part of the case study protocol. These steps are Research Design (1) and Preparation for Evidence Collection (2). Further support of this choice is presented in the next section, as well as a methodology overview, and a summary of Yin's methodology. The third section refers to the design of the research with definition of propositions and units of analysis, and the fourth refers to the preparation for the collection of evidence, including a pilot case to validate propositions and units of analysis. The fifth section present two case studies and their analysis under the proposed protocol. We conclude in the final section with some reflections.

\section{Methodology overview}

Crotty [32] defined methodology as a strategy or process, which makes the choice of particular methods based on the research goals. He also defines methods as techniques or procedures used to obtain data. With regard to methodologies, several authors divide them into three groups [33-35]: quantitative methodologies, qualitative methodologies, and mixed methodologies.

Also called scientific methodologies, quantitative methodologies are normally associated with positivism [33]. Quantitative methodologies are suitable for deductive studies, in which, through the analysis of a representative sample of a population, it is intended to generalize the findings to the population [34]. It is suitable for situations where is intended to test a theory, or identify variables that influence a particular outcome [34] [33]. Examples of these methodologies are experiments [33] and statistical studies [34].

Qualitative methodologies are associated with a constructivist approach, and are aimed to the study of the subjective meaning of events, by paying attention to the views of the participants, their interactions, and the context of events [33]. These methodologies usually are based on the analysis of data in the form of text, obtained for example, through questionnaires and interviews, observations, ethnographic studies, conversations analysis, or meta-analysis [35]. The purpose of these methodologies is to induce theories or patterns of meaning of the experiences [33]. These methodologies are appropriate for exploratory studies, or studies in which the variables involved are not known, usually there are few situations studied and/or situations where the existing theories do not apply [33]. Examples of these methodologies are case studies, ethnographic studies, grounded theory, action-research [33;36], among others.

In addition to these two groups of methods, mixed methodologies are also distinguished currently [33-35]. These methodologies use methods common to qualitative or quantitative methodologies to analyse numerical data and data in textual form [33]. They are useful in cases where, with quantitative methods allied to qualitative ones, it is possible to better explain a phenomenon [33].

To clarify how the nonverbal communication characteristics of 3DVW can influence collaboration, we require data collection via observation and analysis of collaborative situations (in 3DVW) in which these characteristics are common. There are very few studies with this focus. Also the subjectivity of nonverbal communication intrinsic to the context of each situation, and the expected complexity in the analysis of them, led us choose a qualitative methodology: the case study. It relies on the study of concrete situations, not ongoing situations like ethnographic studies, grounded theory, or action research. Observation is our method of choice to collect data, particularly looking at group behaviours, but it could be complemented by interviews or questionnaires as well, in order to obtain more detailed data. Thus we have chosen to employ the case study methodology.

The case study methodology studies phenomena, processes or behaviors in their real environment [31; $39 ; 40]$. It allows the study of different aspects of the object of study and their relationships [40], namely the "how", the "why", and results [39;41]. This methodology is used to explore processes or behaviors that are new or poorly understood [42], characterized by a non-evident distinction between phenomena and context [31], or situations where it is very difficult, if not impossible, to use other methods besides qualitative ones [43]. Techniques of data analysis for case study may include distribution of data by different categories, creating flow charts or other synoptic, calculation of frequencies, means and variances, and organizing data chronologically [44].

In $3 \mathrm{DVW}$, features such as gestures and emotions are used to produce behaviors reflecting social symbolisms like culture, ethnicity, and religion [6-8]. The environment is also used to influence the mood and humor of the users, helping them socialize [6-8]. These behaviors are complex in nature, and are difficult to separate from context, being the spatial environment a good illustration of that fact. These reasons led us to choose the case study methodology for this study. 
We follow Yin's [31] perspective on the case study methodology, which is well accepted and used in many case study research efforts. It comprises the following activities:

- Research Design

- Preparation for Evidence Collection

- Evidence Collection

- Evidence Analysis

- Sharing of Results

Research Design begins by defining the issues under study, or in other words, the research questions. After that comes the definition of propositions, which helps focus the study in the core of the case. The third component of Research Design is the definition of units of analysis, that is, the definition of what concretely will be studied (the phenomenon, behavior, process, etc.). After this, the logical connection of propositions to the data should be made. Finally, the criteria for interpreting the results are defined. After the research planning, the next step is the Preparation for Evidence Collection (or data collection preparation). This implies developing a protocol for the case study - a tool that helps assure reliability in data collection. It includes objectives, framework and relevant literature, procedures for obtaining the data (access, method of collection, calendar), questions to ask, and format of the report with the results. The development of the protocol should be validated with a pilot case. These are the activities presented in this paper. The subsequent activities (evidence collecting, evidence analysis) are accomplished in the pilot case, as well as in two cases afterwards.

\subsection{Methods}

Whatever the research methodology chosen, it is necessary to collect data for further analysis. Thus, the researcher will have to resort to the methods specified in the methodology chosen, which are varied. In this section we present some of the most common methods of qualitative methodologies: observations, questionnaires, and interviews.

Questionnaires are usually used for collecting data that cannot be observed. This method can be anonymous, and can be performed using electronic means or paper [35]. The questions may be open, letting the participants to respond freely, or closed, that is, multiple choices, leaving considerable less freedom of choice. The results of this method are highly dependent on the honesty of the participants, and, furthermore, it tends to be very time consuming.

Observations allow the researcher to obtain information about the behavior of groups of individuals [35]. An observation protocol should be used, to organize the behaviors under observation, and highlight the activities relevant to the research.

Interviews are useful to obtain detailed data on opinions or behaviors, explore new subjects, contextualize data, or provide a more complete description of events [37;38]. However, interviews can be intrusive to the respondents, and may suffer from biases either by the researcher or by the respondents. They can turn into a long process, and usually the results are not adequate for generalization [35;37;38]. According to Olds et al. [35], interviews can be conducted face-to-face or through technological connection, but without anonymity, which makes it a difficult method to use on sensitive issues. The preparation of interviews can be structured, nonstructured, or semi-structured [35;37]. The structured interview follows a closed plan of issues, not adaptable to the operation of any topic that arises unexpectedly. An unstructured preparation begins with a pre-defined issue, and proceeds with questions aimed at discovering an interesting topic to explore. A semi-structured interview has a plan with pre-defined questions, but allows the interviewer to explore some interesting topic that arises.

\section{Research design}

In this work, the research questions are concerned with how nonverbal communication affects collaboration in 3DVW. This general concern can be specified as two questions:

RQ1 How does the use of an avatar influences collaboration in 3DVW?

RQ2 How does the virtual spatial environment influences collaboration in 3DVW?

As for the definition of propositions, in this study they are related to expectations generated by the theory of Presence [15]. Thus, we propose the following set of propositions related to nonverbal communication and the impact it may have on collaboration, based on expectations from previous research on virtual worlds [15]:

P1 The aesthetics of the avatar influence the perception by others of the role of the avatar's user and/or his attitude.

P2 The gestures and sounds that the avatar does influence the perception by others about how the avatar's user wants to collaborate or how he or she wants others to collaborate.

P3 The eye gaze/face direction, direction of movement, and avatar placement provide cues about what the user is paying attention to, or to what the user would like to direct others' attention towards. 
P4 Interaction of the avatar with specific objects provides cues about which objects are intended to be used by others in the collaboration process.

P5 The arrangement of objects (e.g., their grouping or alignment) provides cues of their purpose for collaboration.

P6 The exchange of visual artifacts (i.e., "objects", "clothes", "tools"), with specific visual features and explicit purposes, helps define the team, contributing to group awareness and perception of collaboration roles.

P7 The virtual spatial environment, including lighting, sound or music, and visual effects, influences the attitudes of collaborators.

We also defined the units of analysis both for avatars and for the virtual spatial environment. Referring to the research questions, the units of analysis for avatars we used were:
- Appearance
- Gestures made
- Sounds emitted
- $\quad$ Eye gaze
- Facial demeanor
- Facial orientation
- Direction of movement
- Body position
- Avatar placement
- Visual artifacts used for interaction

And the units of analysis for the physical space were:

- Animated visual artifacts (animated objects) or artifacts for interaction (i.e. pose balls)

- Non-animated visual artifacts

- Non-visual artifacts (e.g., scripts)

- Visual environment (e.g., what kind of place the action is taking place in)

These units of analysis are the observation targets of the case studies, and their relations with the research questions will be obtained by applying Yin's recommended criteria [31].

\section{Preparation for Evidence Collection}

In the preparation for evidence collection, a protocol for the case study should be developed, and tested by a pilot case. In the first subsection the protocol will be summarized, and then a pilot case will be presented to validate the protocol, and to clarify the usage of the protocol.

\subsection{Protocol for the case study}

As a general objective, the case studies are supposed to clarify the relationship between nonverbal communication and collaboration in 3DVW. Thus, two goals for the studies can be stated: to clarify the influence of the avatar in collaboration 3DVW; and to clarify the influence of the virtual space in collaboration 3DVW.

Data collection is based on the unit of analysis presented on the second subsection. The collected data are compiled and related to each one of the units of analysis. They also have to be related to the propositions by creating logical chains of evidence. These chains should lead the reader's reasoning to the evidence that there is a relationship between the data and the proposition in cause. So, every proposition is assigned to one or more evidences related to the subject of the proposition. These elements are the beginning of each of the chains, and then added to other evidences, also related to the proposition, to complement the chain.

To clarify how this analysis should work, we present the following example: suppose that, in a given case, the following data was collected: Element 1 - "the avatar raised his arm in a certain direction, while looking to the visiting avatars"; Element 2 - "the same avatar said «follow in this direction»"; Element 3 - "the others followed the direction pointed by his arm". The first element may be directly related to gestures, a subject of Proposition 2. Element 2 chains with element 1 leading to understand why element 3 was observed, i.e., the other users realized that by showing his arm, he was indicating them to follow that direction, confirming proposition 2 .

For each case studied, a report shall be prepared. This report shall contain the following elements: case identification; summary description of the case, if the source is documental, otherwise description of the collaborative situation, scenery, actors, and other relevant facts; the narrative of the case, again if the source document is, if not, a description of the observed facts shall be presented, preferably with examples such as images, messages exchanged, etc; the data compilation and analysis shall be presented in tables (such as those presented in the next cases).

\subsection{Pilot case}

Second life is being used for collaboration in several different tasks, with learning and training as one of the most common [15]. This case was selected for convenience, because we had easy access to it for observation. In the pilot case used, the data was obtained by direct observation. The pilot case is an example of collaboration on an initial training class, where new participants of a group in Second Life learn the basics of building. The group's theme is the Star Trek television series. The group has several activities, among which 
stands out the construction of objects related to the series, with particular emphasis on the production of spaceships. Thus, it is of great importance for the group's goals to teach newcomers how to build a variety of objects.

\section{Scenario}

For the scheduling of the class, a Second Life group notice was used with date and time (this is a typical text message that is broadcast to all group members). The class was held in an empty space, commonly called "sandbox" in the context of Second Life (regardless of whether it has any actual sand or whether it is an actual box or - most likely - not), large enough to build even space stations. This sandbox space had many participants moving around, positioning themselves close to some of the objects, and often facing them. It was possible to observe beams of light balls coming out from the hands of some of the avatars towards some of those objects. These are Second Life's cues to indicate that an avatar is editing an object, so it was no surprise that the presence of those beams coincided with striking visual changes in the objects to which they were emitted.

The class consisted of several avatars, dressing uniforms and bearing titles visible as text hovering above their heads, identifying them as several cadets, two junior officers who constituted the instruction team, and a senior official responsible for the supervision, as explained by one of the instruction team members. Participants unaware of the significance of titles and uniforms could check them in a text file, which alongside others (with rules, schedules, activities, etc.), as well as uniforms, titles, and other objects, are available to group members at a dedicated warehouse. Usually, these resources are informed to newcomers by a host.

\section{The class observation summary}

As soon as all participants gathered around the instruction team, forming roughly a circle, the instructor used the voice channel to present himself, welcoming everyone, and to transmit certain operating rules for the class. Besides rules, the roles of each instruction team member were also transmitted to the group, as well as a summary of the program for the class. The instructor offered to explain while demonstrating, and began to do so. While explaining, an object came up in front of him. A beam of light balls coming out of his hand towards the object pointed out he was editing it, and indeed changing as mentioned by the instructor. Students emitted similar beams towards objects that appeared before each of them. This indicated which object each one was editing, and those objects started to change shape as the instructor's had. After explaining using voice communication how to control the most basic properties such as shape, position, and dimensions, and a few others, the instructor started to talk about the control of color and texture of objects. He mentioned that he would render a chair, and a chair appeared in front of his avatar, after which he urged the group of cadets to make an equal one as an exercise. He also said he would distribute a texture to be used in the chairs, using Instant Messaging (IM) as a means of distributing the resource containing the actual texture. Several objects came up on the ground near each other, as a sort of grouping, before each student. Again, beams of light balls were emitted from the hands of the avatars towards the objects that began to change shape, position, or texture.

Further along in the class, the overall position of those groups of objects relative to each other revealed them to be chairs similar to the instructor's. Sometimes, some students issued messages in text chat, or made their avatars start animations/sounds such as waving and whistling. Upon occurrence of those animations or sounds, the instructor and assistant would address the source avatars, communicating by voice. It was observable that sometimes from the hands of the avatars of the instructor team, beams of light balls would come out again towards the objects in front of the students, changing them. After everyone finished the exercise, with varying degrees of success, the class was declared ended by the instructor.

\section{Evidence collection in the pilot case}

The evidences were drawn directly from the description of the above case. Thus, each reference to the use of a feature or behavior was accounted as evidence, relating it to one or more units of analysis, according to the impact of the evidence described in the unit. Not all of the units of analysis have evidences in this case. Table 1 summarizes the evidences of the units of analysis for the avatar, with a brief description of the reference of the case description. Similarly, Table 2 summarizes the evidences of units of analysis for the physical space.

\section{Evidence analysis in the pilot case}

To analyze the collected data, several evidences were related in order to create a chain of evidences to support each of the propositions mentioned above. Table 3 summarizes the propositions and the chains of evidences supporting them. Each proposition has one or more chains of evidences, each beginning with an evidence of a unit of analysis directly related to the proposition, as shown on Table 3. The other elements are presented in a lower layer, and are connected with arrows. These elements of the chain may or may not be from the same unit. In some cases, evidences taken directly from the case were added in a third layer, to help clarify the relationship.

The first proposition related the appearance of the avatar, is supported by the fact that all participants' avatars wear uniforms. The meaning of the different uniforms is available either textually or verbally. 
Referring to gestures and sounds, the second proposition is sustained by the reaction the instruction team had in assisting the students, when some of them used gestures and sounds, sometimes accompanied by messages in chat.

The proposition on the direction of movement, eye gaze/head direction or avatar placement is supported by two chains of evidence. The first is related to the movement of other avatars who do not participate in class, but their physical attitudes, gestures, and interaction on objects, reveal their activities. The second chain starts at the reunion of students around the instruction team, which triggered the beginning of the instructor's exposition.

The following proposition, related to interaction with objects, is also supported by two chains of evidence. The first is based on the object used by the instructor to reflect the intentions expressed by him, leading students to imitate his actions. The second, it is based on the exercise proposed by the instructor, which urged the students to build a similar chair to the one presented.

The next proposition, about the arrangement and grouping of objects, is based on the fact of the objects that students have in groups near them, having their properties changed to form chairs. This fact is so revealing of completion that the instruction team, when addressing some participants to assist them, used some of these groups of objects for assistance of others.

The proposition related to the exchange and use of objects and artifacts is sustained by three chains of evidence. The first is related to the fact that the participants have gathered at the date and time scheduled by a group notice. In the second chain, the titles clarify each participant's role in the group. And in the third chain the texture's function confirms the intentions of the instructor.

Finally, the proposition concerning the environment is supported by the fact that the class has taken place on a site whose purpose is to render and build objects. This purpose is supported by its visual properties: being large and empty, perhaps with a few scattered disorganized artifacts resulting from previous building exercises, which for users of Second Life are all cues enabling the identification of the space as a "sandbox".

\section{Case studies}

In this section we present two case studies. These cases will be analyzed with the protocol presented in the fourth section. Both of them were held on a school class environment, somewhat similar to the pilot case that was a training situation. This similarity constitutes some continuity in the studies that, we expect, will contribute to some insight about the influence of nonverbal communication in collaboration in 3DVW.

\subsection{First Case}

The first case is an of English language class held in Second Life. The school is called English as Second Language, and provides any number of classes for a fee. The schedule of classes is on a panel on the welcome area (Figure 1), or can be found on the Web in a dedicated page. The activity carried out in this class is a "class excursion", i.e. it is not held in a school classroom, but somewhere in Second Life. The use of voice (audio) communication is required and is used exclusively. Thus, even if this fact is not mentioned, it should be understood that all dialogs take place using this medium. In Second Life, the avatars that have voice enabled have a white ball hovering over their heads. When they speak, green arches appear around the ball resembling an animated volume icon, with more or less arches to coincide with the change in intensity of voice we can hear. With these means we can know who can speak and who is speaking.

Table 1. Evidences related to the avatar (Pilot case).

\section{Appearance}

All participants' avatars were dressed with uniforms.

\section{Gestures made}

Beams of light balls could be seen coming out of the hands of some of the avatars.

A beam of light balls was emitted from the hand of the instructor towards the object of exemplification.

Students emitted similar beams towards objects that came up before each of them.

During execution of the chair-building exercise, light beams where emitted from the hands of several avatars towards objects that changed shape, position and texture.

Beams of light balls would be emitted from the hands of the instruction team towards objects of students, changing them.

\section{Sounds emitted}

Some students made calling gestures and/or sounds such as waving and whistling.

\section{Direction of movement}

Instructor and assistant walked towards the students.

\section{Avatar placement}

The sandbox had several participants moving around, positioning themselves near some of the objects and often facing them. 
Participants gathered around the instruction team, forming roughly a circle.

\section{Visual artifacts used for interaction}

Visible changes of the physical objects near avatars were observed.

The instructor's object reflected the changes mentioned by him.

Objects near students changed in a similar manner to the instructor's.

The instructor rendered a chair.

Objects in apparent groupings in front of each student.

Students' objects changed shape, position and texture.

Light balls were emitted by the hands of the instruction team towards objects of students, changing them.

Objects in front of each student assumed a spatial positioning resembling a chair.

Table 2. Evidences related to the physical space (Pilot case).

\section{Non-animated visual artifacts}

The instructor used an object for demonstration.

The instructor rendered a chair.

Objects appeared in apparent groupings in front of each student.

All avatars had text titles visible over their heads.

\section{Non-visual artifacts}

The scheduling of the class was provided by a group notice with date and time.

Text notes with rules, schedules and activities, are available to group members, as well as uniforms, titles and other objects.

The instructor distributed a texture using IM.

\section{Visual environment}

The class was held on a large empty space ("sandbox").
Table 3. Chains of evidences (Pilot case).

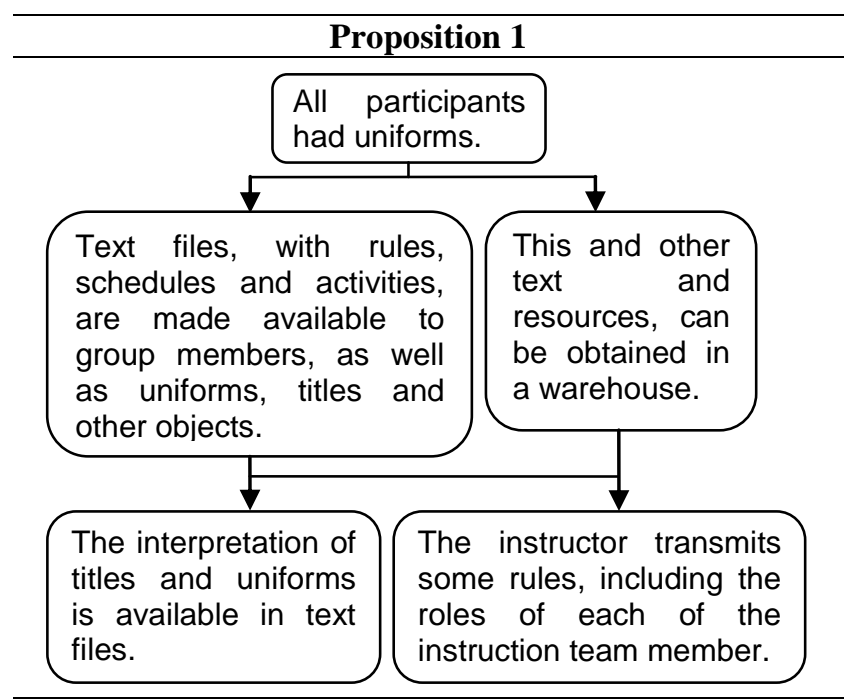

\section{Proposition 2}

Some students made gestures and/or sounds such as waving and whistling.

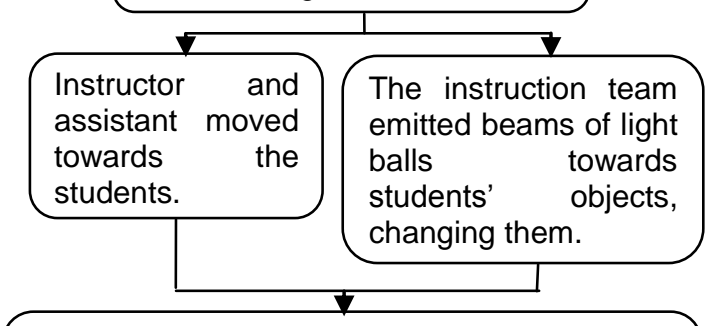

Some students used text chat messages, or made animations and/or sounds, after which the instructor and assistant addressed those students, communicating by voice.

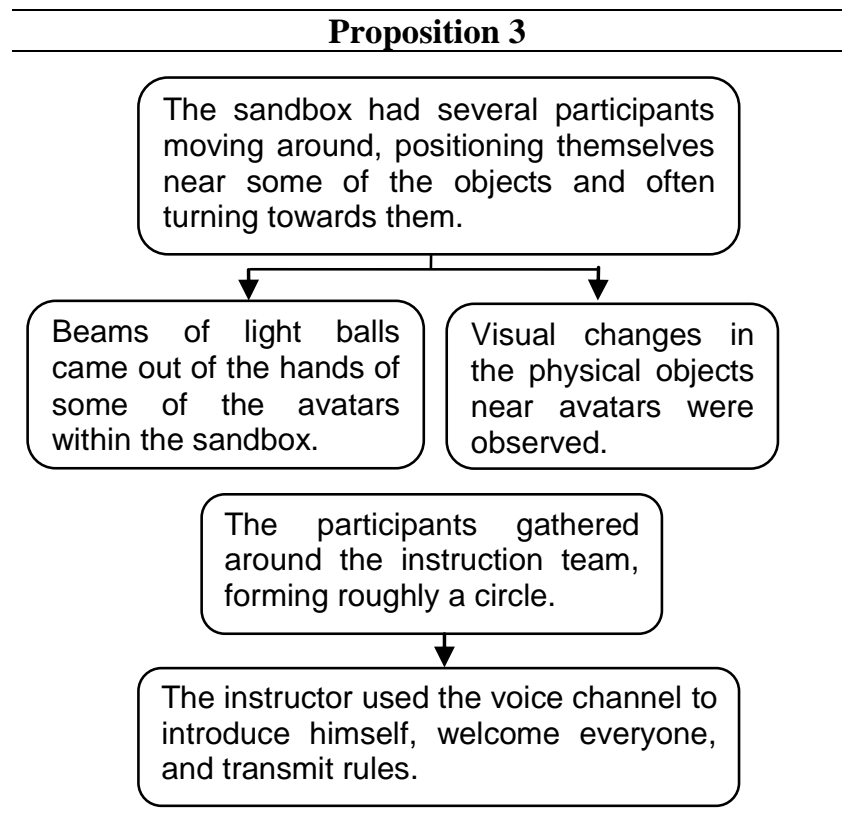



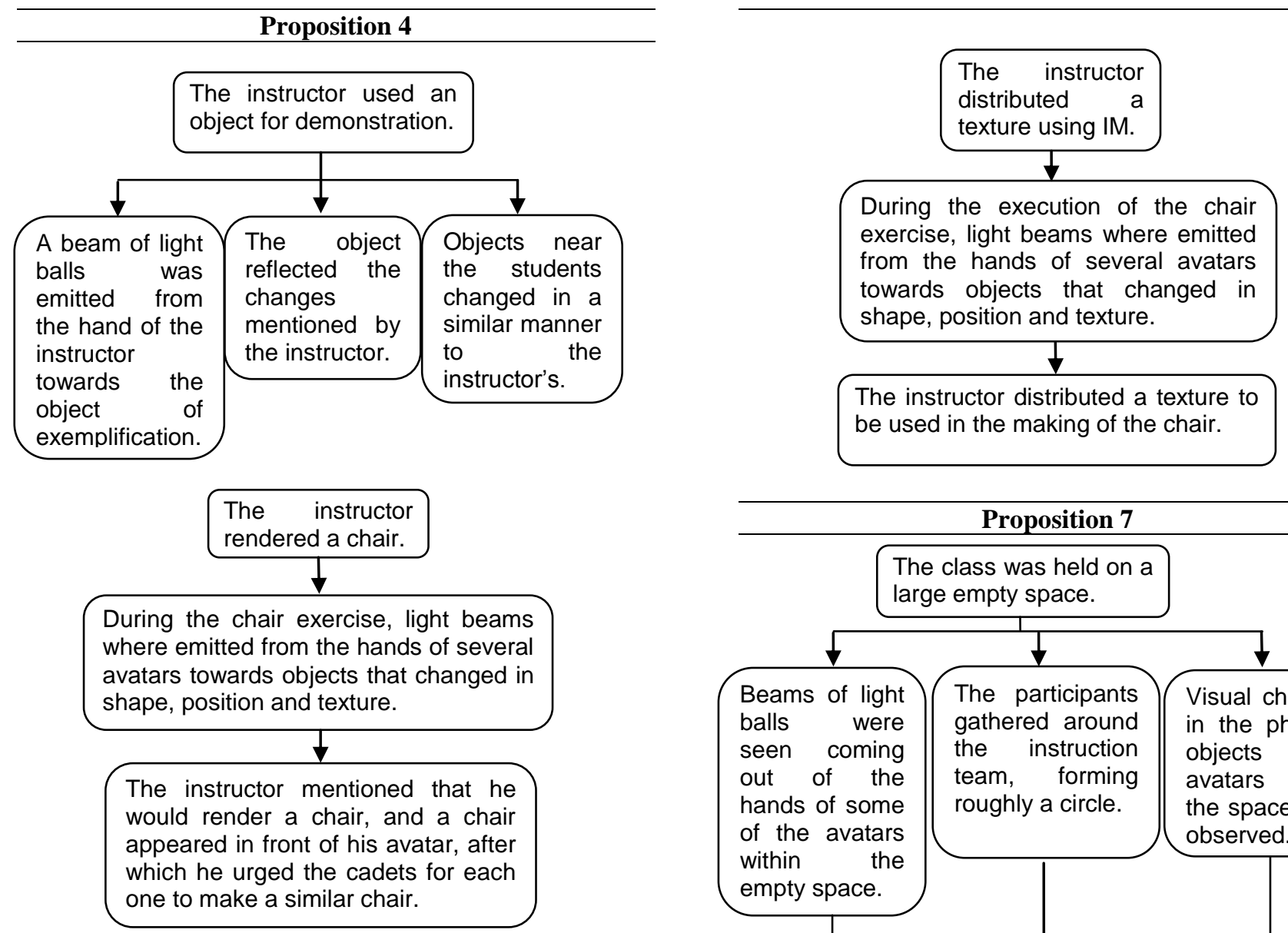

\begin{tabular}{|l|l|l|}
\hline \multicolumn{2}{|c|}{ Proposition 5 } \\
\hline $\begin{array}{l}\text { Tisually grouped objects appeared } \\
\text { in front of each student. } \\
\text { changed objects } \\
\text { position and } \\
\text { texture. }\end{array}$ & $\begin{array}{l}\text { Objects in } \\
\text { front of each } \\
\text { student were } \\
\text { assembled } \\
\text { resembling } \\
\text { chairs. }\end{array}$ & $\begin{array}{l}\text { The instruction } \\
\text { team emitted } \\
\text { beams of light balls } \\
\text { towards some } \\
\text { students' objects, } \\
\text { changing them. }\end{array}$ \\
\hline
\end{tabular}

\begin{tabular}{|c|c|}
\hline \multicolumn{2}{|c|}{ Proposition 6} \\
\hline $\begin{array}{l}\text { The scheduling of } \\
\text { the class was } \\
\text { provided by a } \\
\text { group notice with } \\
\text { date and time. }\end{array}$ & $\begin{array}{l}\text { They all had } \\
\text { titles visible over } \\
\text { their heads. }\end{array}$ \\
\hline $\begin{array}{l}\text { The participants } \\
\text { gathered around } \\
\text { the instruction } \\
\text { team, forming } \\
\text { roughly a circle. }\end{array}$ & $\begin{array}{l}\text { Text notes with } \\
\text { rules, schedules } \\
\text { and activities are } \\
\text { available to group } \\
\text { members, as well } \\
\text { as uniforms, titles } \\
\text { and other objects. }\end{array}$ \\
\hline
\end{tabular}
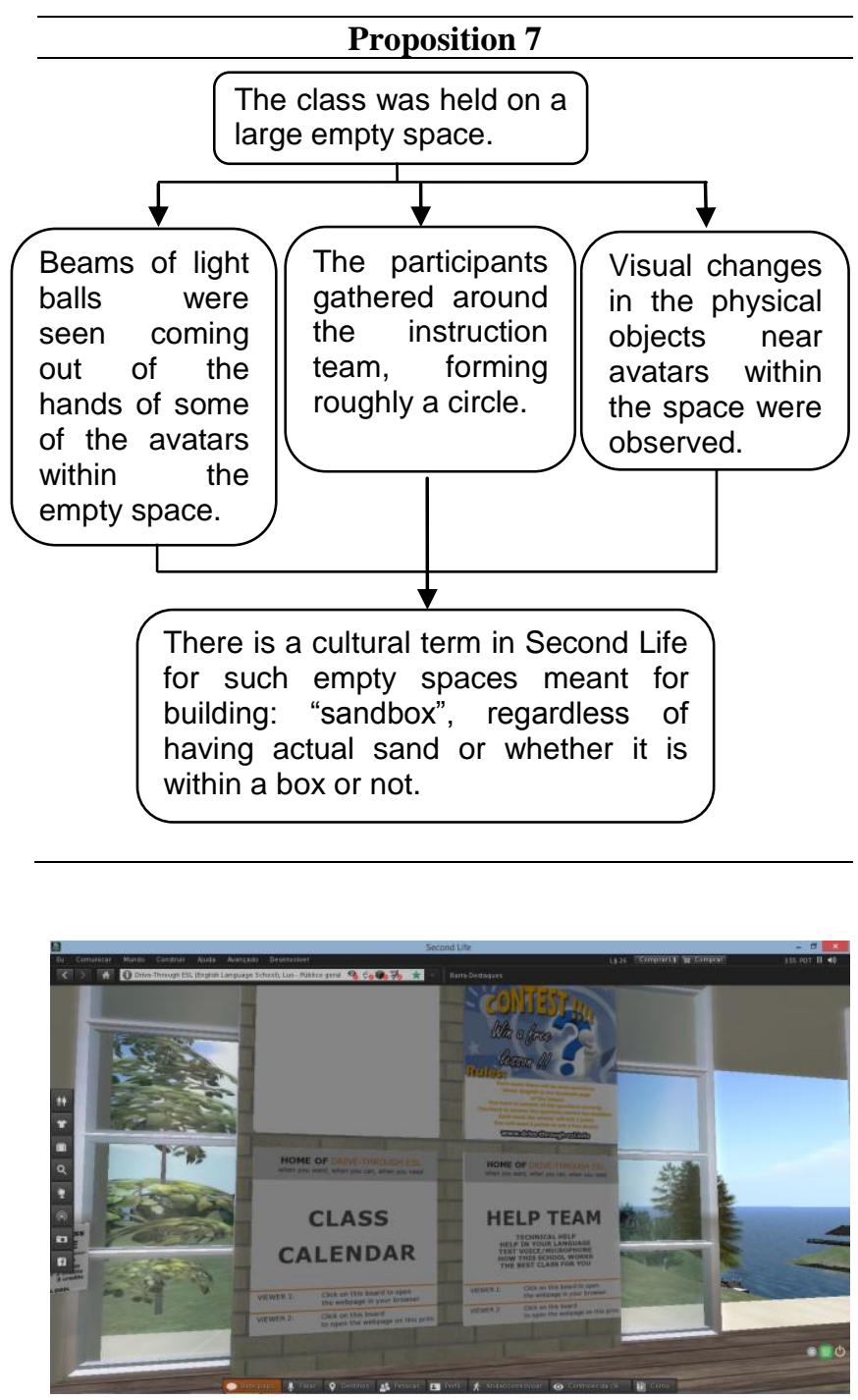

Figure 1 - Welcome area.

\section{Scenery}

This type of classes, the excursion class, is usually held in different places in Second Life. The goal is to ask students to describe what they see on the sites, using the 
English language. In this particular case, the class was held in a place called Art Box. The place has two levels. In the top level there are several pictures, mainly about paintings, but many of them also related to movies, music, and other art forms (Figure 2). The bottom level is completely empty, with a white background. By clicking with the right mouse button on each of the top floor images, and selecting "sit" on the dropdown menu, a three-dimensional representation of the selected image is rendered on the ground floor. These representations have pose balls in front of them that allow avatars to take positions to be part of the scene.

\section{Case summary}

After receiving an IM with the announcement of class and a link, and following the link, researcher's avatar appeared on site described above. On this place, there were three avatars, one male and two female, judging by their appearance. Voices were heard greeting the researcher, to which the researcher replied, greeting them too. The tones of their voices coincided with the gender of the avatars. One, with a text box on the head identifying it as a teacher, asked the researcher to pose with them in a picture that was already in the same place (Figure 3). The theme of the picture was about the pop music group "The Beatles." By clicking on a pose ball in front of the picture, it was possible to replace one of the characters of the scene with the avatar (Figure 4). The other avatars present started to appear in the scene. After selecting "sit" from the last of the pose balls present, the researcher's avatar also replaced one of the characters (Figure 5).

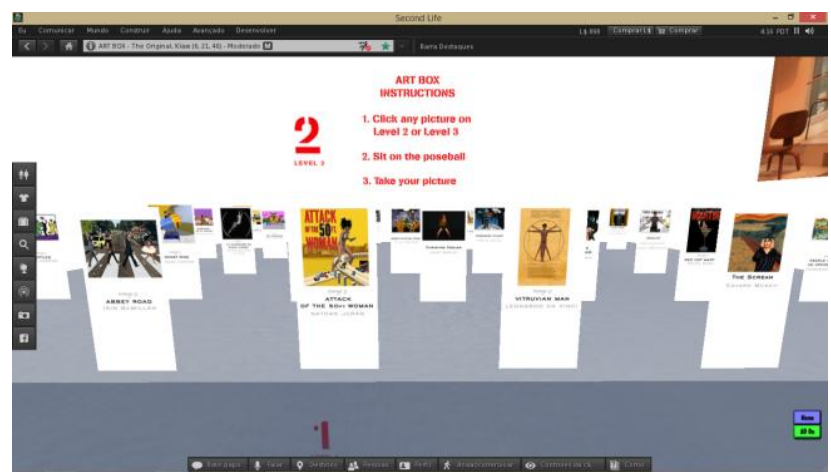

Figure 2. Top level with the images.

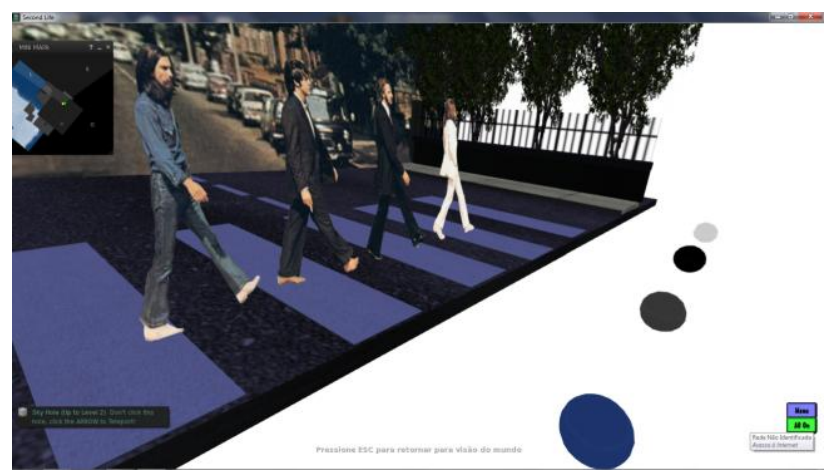

Figure 3. Tridimensional recreation of the image of the pop group "The Beatles".

The teacher then began to ask questions about the scene. The voice bars were clearly visible over head the teacher's avatar. Then, she abandoned the pose and walked towards a black circle on the floor, positioning herself near a blue arrow hovering over the ground, pointing to the circle. Then, she told a male avatar to follow her, in order to choose another art object. To do this, she told him to "sit" on the blue arrow. The teacher's avatar directed her face to the arrow (Figure 6). Then, after some light orbs hovered around the arrow, the avatar of the teacher made an entry animation in the circle (Figure 7).
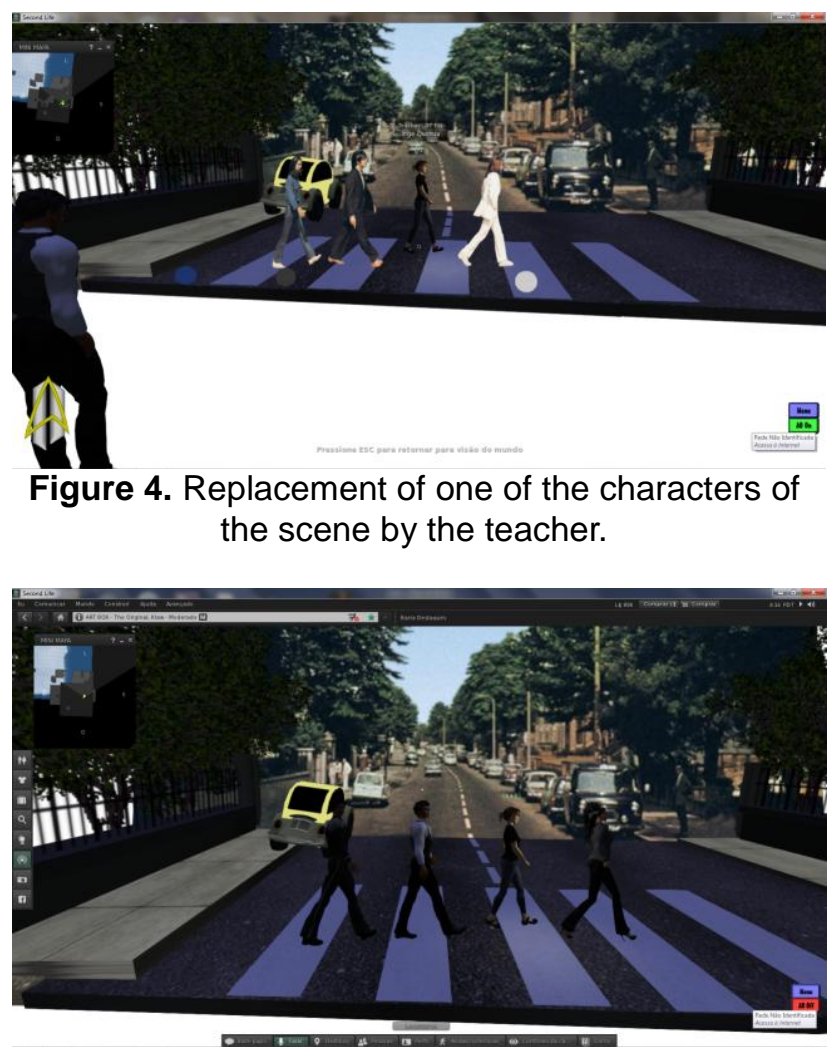

Figure 5. The avatars replace all the characters of the scene. 


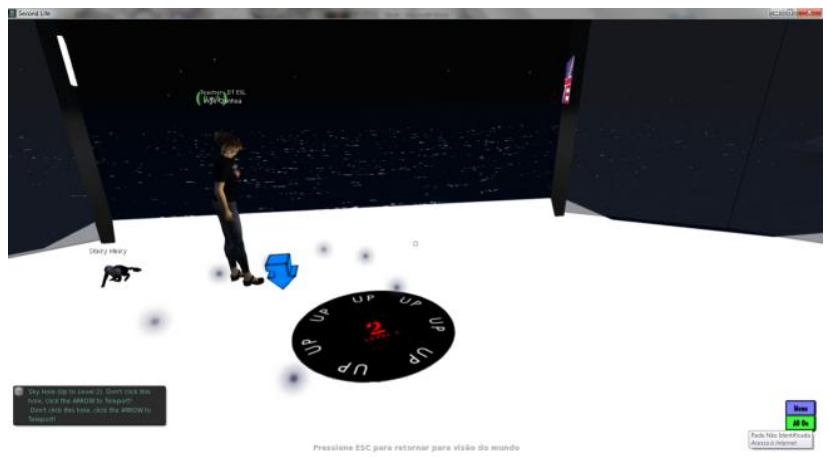

Figure 6. Arrow to transport avatars between levels.

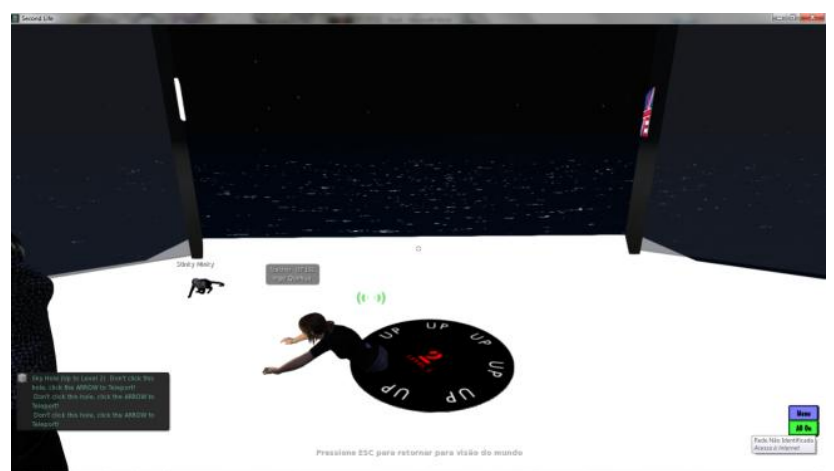

Figure 7. Animation associated to the transport.

The male avatar followed her showing the same animation. A few seconds later a new three-dimensional scene appeared (Figure 8). The teacher and the student came back. The teacher asked the student to describe the scene. While the male avatar described the scene, the voice's over his head was visible. This scene had two pose balls, one of which the teacher used, appearing on the scene. After the description of this scene, the teacher asked the researcher to follow her through the circle as described above. The researcher followed her and went to the upper level. Then, she asked the researcher to select an image. The researcher did so by selecting "sit" from one of them (Figure 9). In the lower level appeared the corresponding three-dimensional scene to the image that had been selected (Figure 10).

The teacher touched the only pose ball available on the scene, and became part of it. Then, she asked the researcher to describe the scene, which he did by voice. After the description, she asked a female avatar to follow her, and she did so. A few seconds later, another scene was rendered replacing the previous one (Figure 11). This scene had several pose balls. After returning to the ground floor, the teacher asked the female avatar to describe the scene, and she described it. The teacher's avatar positioned itself next to conical objects that are part of the scene, and she asked what that could be. It was visible that her avatar was directing the face towards the objects (Figure 12). The other female avatar positioned itself next to the teacher, and directed its face to the object too. Then, a beam of light balls became visible, coming out of the teacher's avatar hand towards the conical object. After that, the teacher went to the top floor again, and again another scene was recreated in the lower floor. The teacher said that this place was very interesting, and that she would return there with other classes, but this class was over. So, everybody said goodbye and started to disappear. The teacher said goodbye by text chat as well (Figure 13).

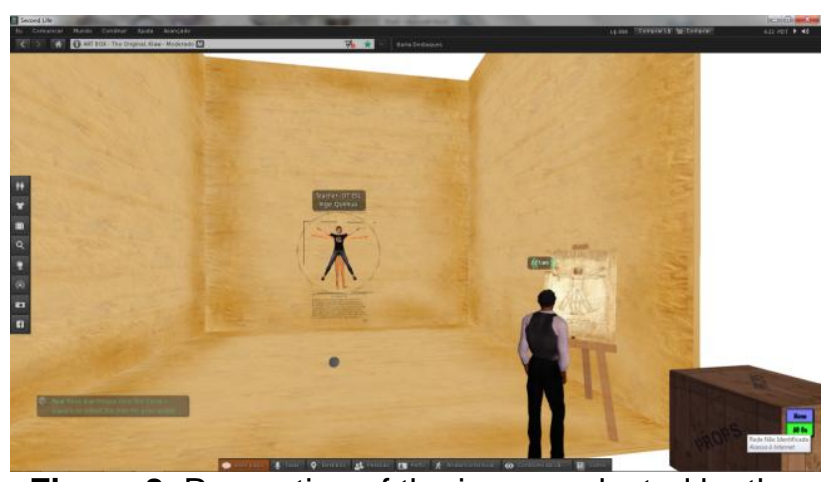

Figure 8. Recreation of the image selected by the other male avatar.

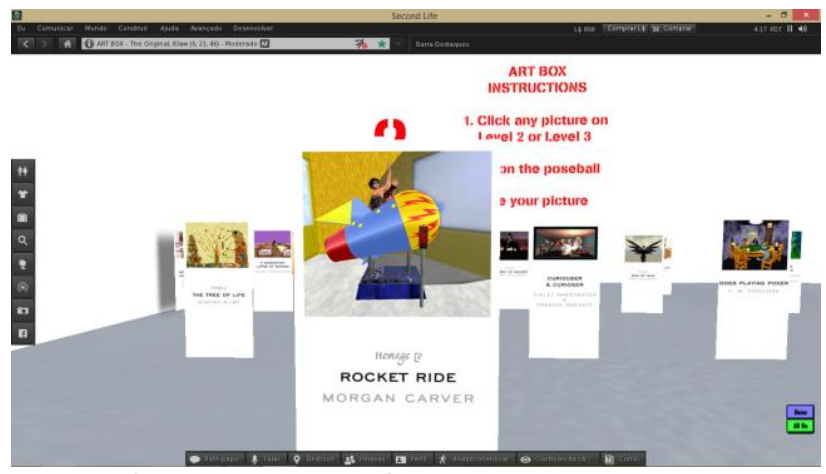

Figure 9. Detail of the Picture selected.

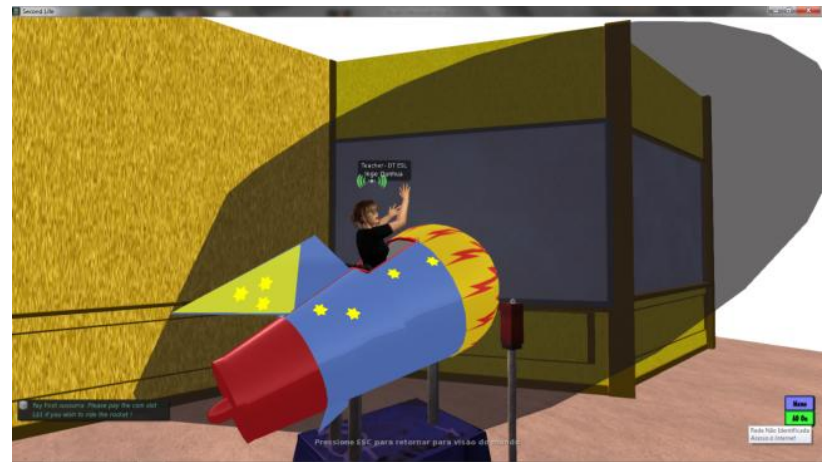

Figure 10. Recreation of the selected picture. 


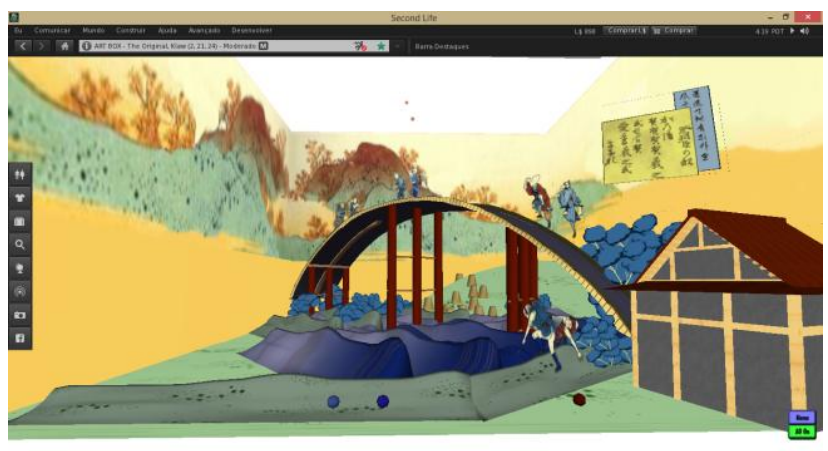

Figure 11. The scene chosen by the female student.

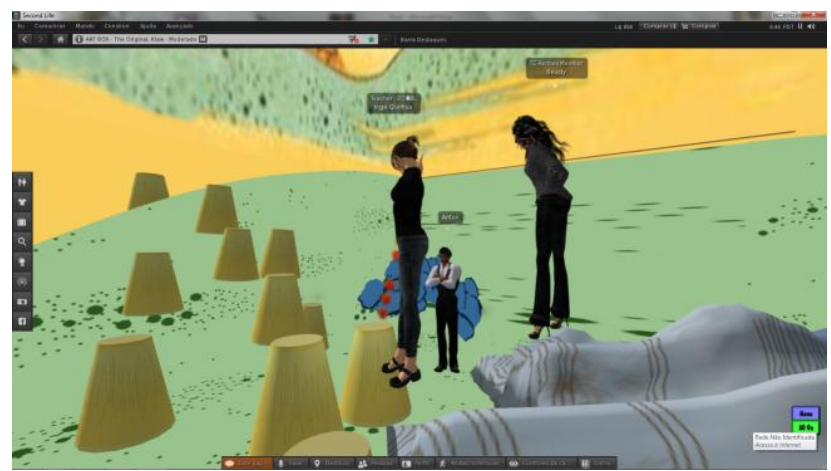

Figure 12. Detail of the conic objects.

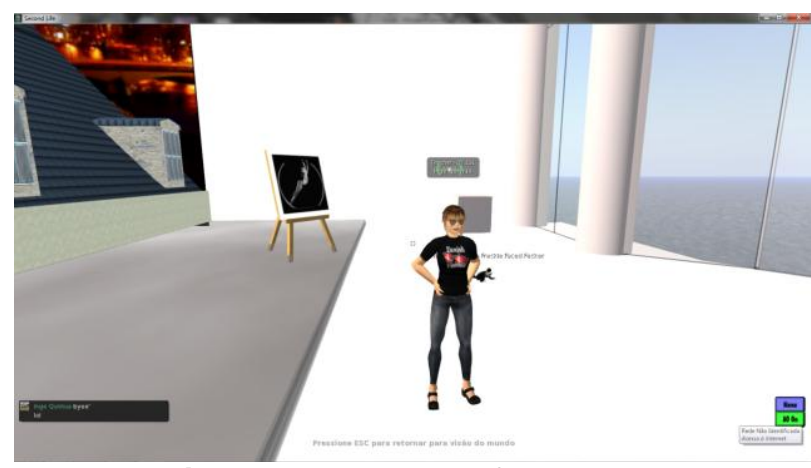

Figure 13. The end of the class.

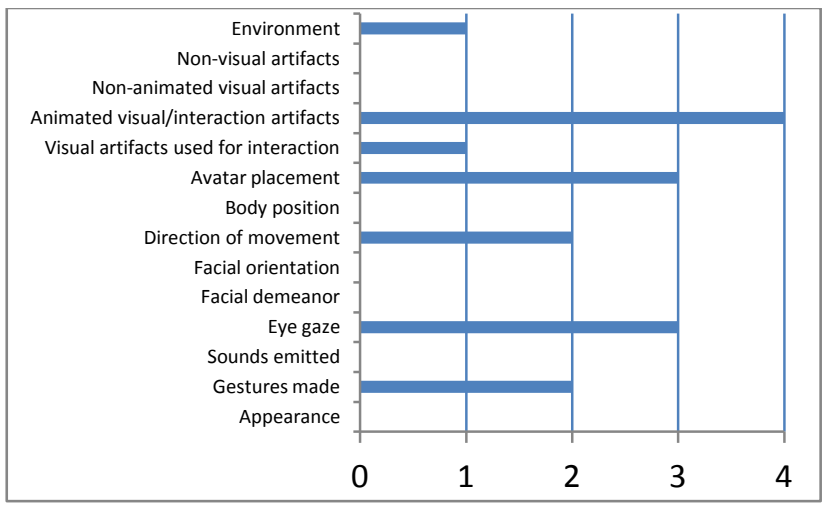

Figure 14. Distribution of evidences throughout the units of analysis (Case 1)
Table 4. Evidences related to the avatar (Case 1).

\section{Gestures made}

The avatar of the teacher made an entry animation in the circle.

The male avatar followed her revealing the same animation.

\section{Eye gaze}

The teacher's avatar directed the face to the arrow.

It was visible the avatar of the teacher directing the face into a conical object.

The other female avatar also directed to face the object.

\section{Direction of movement}

The avatar of the teacher is directed to an existing black circle on the floor positioning itself near a blue arrow hovering over the ground.

The teacher's avatar positions next to conical objects that are part of the scene, and she asked what those could be.

\section{Avatar placement}

The avatar of the teacher appeared in the image by replacing one of the characters of the scene.

The other avatars present did the same.

The other female avatar is positioned itself next to the teacher.

\section{Visual artifacts used for interaction}

The voice icon over the heads of the avatars.

Table 5. Evidences related to the physical space (Case 1).

Animated visual artifacts (animated objects) or interaction (pose balls, teleport objects, etc.)

Text box identifying the role of teacher.

Pose balls.

Black circle on the floor and blue arrow.

Art images.

A beam of light balls was visible coming out of the teacher's avatar hand towards the conical object. 
Visual environment

Empty space with white background.

\section{Evidence collection}

In this case 17 pieces of evidence were collected, 11 related to the avatar, 6 to the physical space. The distribution of elements by the units of analysis is as follows (Figure 14): "Animated visual artifacts or interaction" had 4 evidence; "Eye gaze" and "Avatar placement" had 3 elements each; "Gestures made" and "Direction of movement" had 2 each; "Visual artifacts used for interaction" and "Visual environment" had 1 evidence each; and there were no other elements. These elements are compiled in Tables 48 and 5.

\section{Evidence analysis}

In this case the first proposition has no chain of evidence. The second proposition has two chains of evidence. The first one starts with the teacher exemplifying how to go to the next floor, and then, she is followed by the avatar she had called. The second chain of evidence also starts with the example of a teacher, when she poses like she wants' others to do. The third proposition has three chains. The first refers to the circle used for teleportation. The teacher's avatar walks to the circle and moves the head, turning its face to the blue arrow. That head movement follows the movement of the user's mouse, which may be revealing of where the user is looking or to what is the focus of attention. This is a way of reinforcing the intention to direct the attention of others to the arrow. The second chain refers to the same movement of the head revealed by the teacher, but this time towards one of conical objects. This interpretation of the look is seconded by the beam of light balls coming out of the hand of the teacher's avatar towards the same object. Such beams appear when someone tries to edit an object in order to know more details of it, or to change it. In the third chain of evidences to other female avatar reveals the same head movement towards the conic object, after positioning the avatar near the teacher. The fourth proposition as a chain of evidences that starts with the role of the teleportation used to go to the upper floor. By using it, the teacher makes it clear her intention is to be followed. The fifth proposition also has only one chain related to the positioning of the pose balls right in front of the scenes, drawing attention to them. The sixth proposition has also only one chain of evidence related to the text box hovering over the head of the teacher's avatar, identifying it as a teacher. That induces participants to follow their example and participate in the activities without discussing or questioning the legitimacy of the teacher, because she is clearly identified. The last proposition, also with only a chain of evidence, refers to the empty space, implicitly prone to be filled by re- creations that participants choose, and are used for the activities of the classes. All these chains are summarized in Table 6 and their distribution throughout the propositions are presented in Figure 15.

\subsection{Second Case}

The second case is also an English language class held in Second Life, of the same group. This time it will be held in a traditional classroom. It also required the use of voice and used it exclusively. So, again, it should be understood that all dialogs are using this medium.

\section{Scenery}

The class took place in a room with simple furniture and decoration (Figure 16), similar to a real classroom. It has boxes arranged as in a theatre, used as stools. On the opposite side there was a small stage near the wall, and in that, a panel with the school symbol.

\section{Case summary}

Minutes before the beginning of the class, the researcher doing the observation received an instant message (IM) from his school contact. This contact is recorded on the calendar as being in charge of the class. The IM told of the beginning of the class, and it had a link. The link led to the school arrival area. In there was the avatar who contacted the researcher and two others.

Several voices could be heard and voice icons were visible over their heads. The avatar responsible for the class said that one of the avatars was being heard loud and clear. Then, he greeted the researcher and asks if the researcher could speak. The researcher answers yes, and greeted all people present. The avatar responsible for the class asked the researcher if he was being heard well, and he answered yes. He said he was hearing adequately on his side too. Then, he told all present to go to the classroom. He asked the group to follow him and moved towards a gray circle on the floor. He positioned himself facing the circle, while close to it. Then he told us to touch the circle and, after his avatar assumed a sitting position on the circle, it disappeared. Touching the circle the researcher's avatar assumed a sitting position on the circle, and then appeared on a similar circle in another room (such objects are known in Second Life as "teleporters"). Looking around, turning the avatar from one side to the other, the researcher could see the room and the teacher, as well as other avatars that had been arriving, and began appearing on the gray circle too (Figure 16). 
Table 6. Chains of evidences (Case 1).
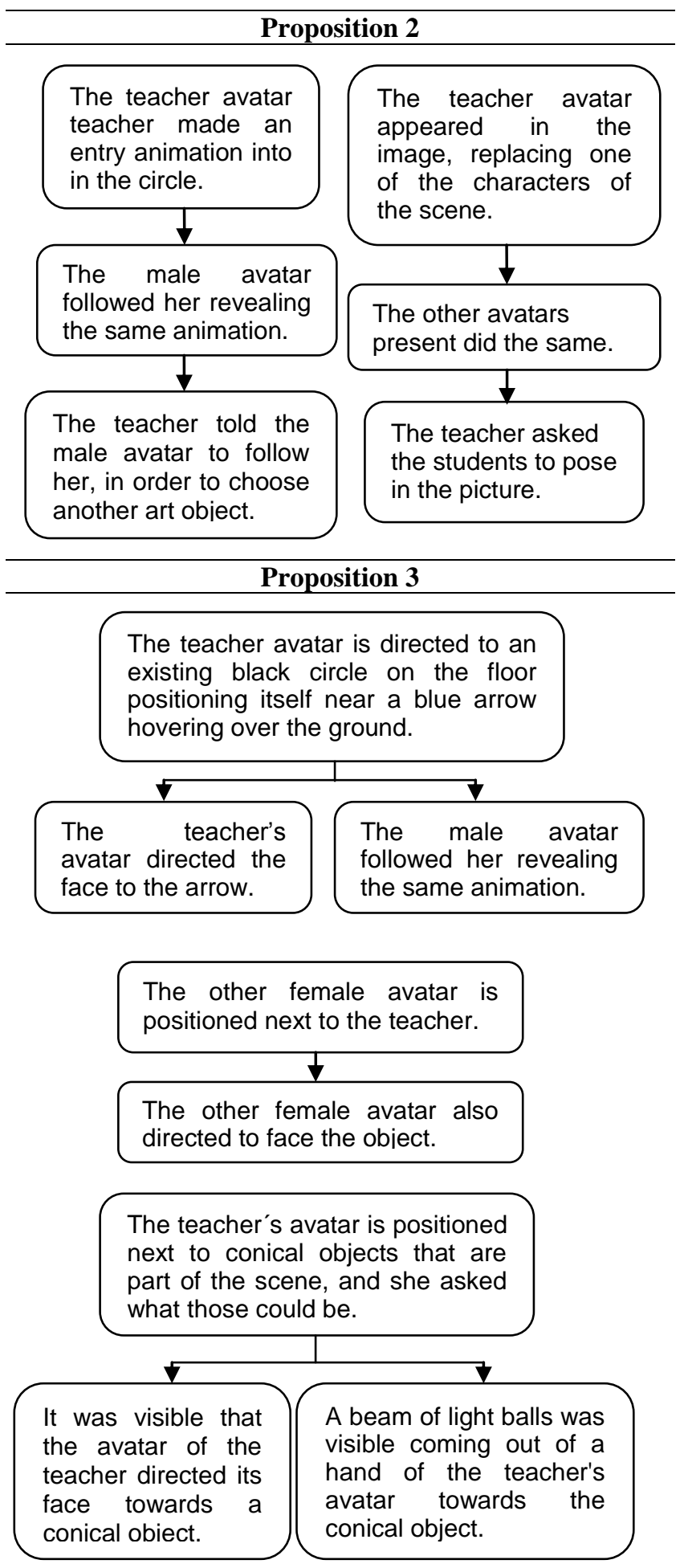
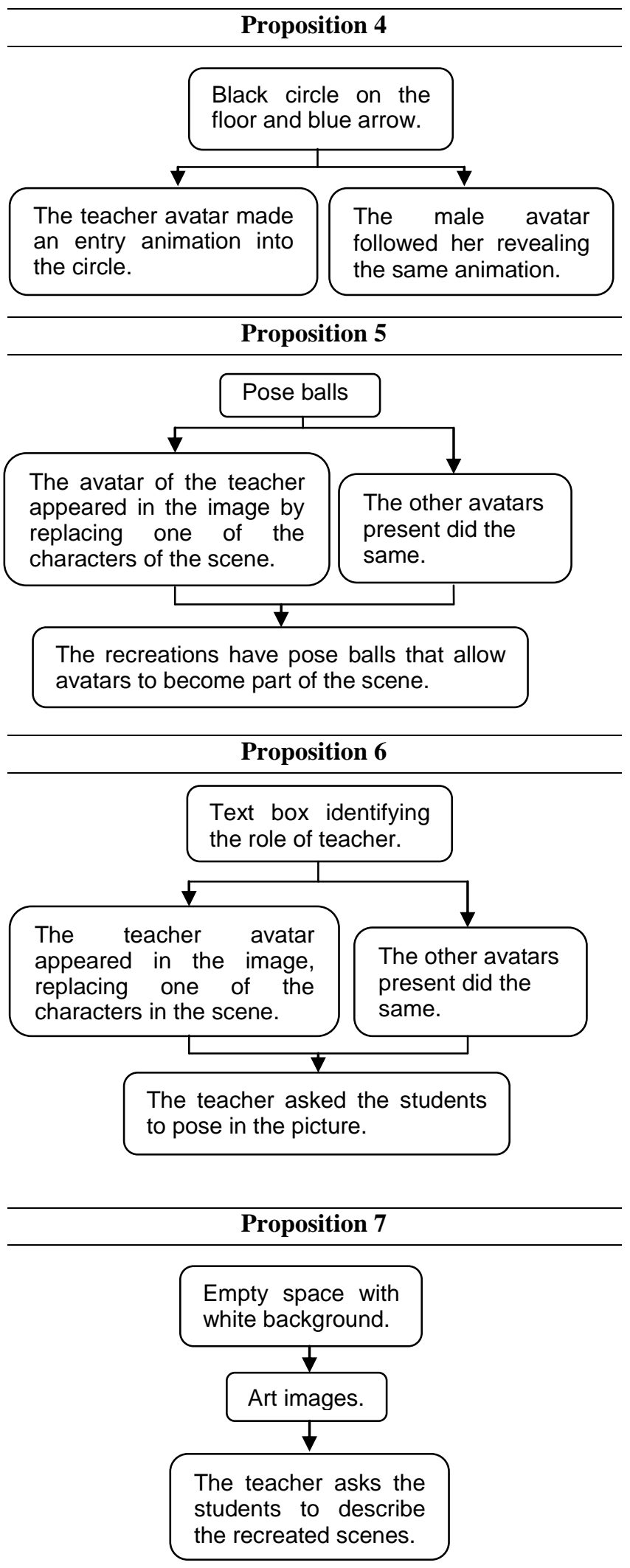


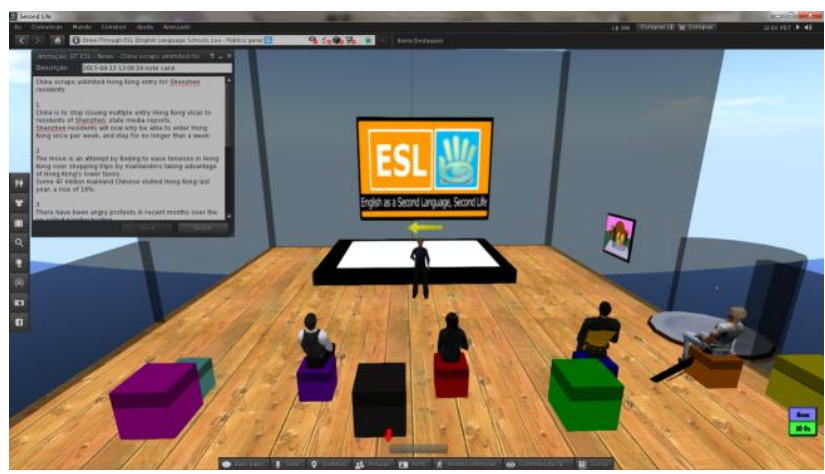

Figure 16. Classroom interior. The teleporter is on the right side of the room.

The avatar responsible for the class asked the group to sit. The other avatars entered the room and sat on the boxes in the front row, facing an image across the room. The researcher sated his avatar on one of the boxes available, by positioning the mouse over it, opening the menu with the right button and selecting "sit". The researcher's avatar assumed a sitting position facing the image too. The avatar responsible for the class disappeared. After a few minutes, he was back. The image on the wall across the room was replaced by a school billboard. The avatar responsible for the class was standing, positioned next to the stage, with his back to the billboard. Thus, he was facing the other avatars. Meanwhile, another avatar appeared in the gray circle on the floor. After greeting each other, the responsible asked him to sit, and he sat in the front row, on the nearest available seat.

The teacher began the class by voice. The researcher got from him a note-card with a text in English. He explained that he would read the whole text, and then each student would read a paragraph. He read the text, and then asked everyone present to read a paragraph. The voice icons were visible over the head of each avatar has he was reading, and reading could be heard. The teacher interrupted the reading occasionally, correcting a word or expression. The reader repeated the word or expression attempting to pronounce it as the teacher had done.

After each reading, the teacher asked if anyone had any questions. Some attendees said they did not understand what some words or expressions meant, their voice icons clearly visible while they did that. The teacher explained those words or expressions. At the end of the answers, he asked again if the students had understood the explanation. After reading all the text paragraphs, the process was repeated with a second text in a second notecard sent by the teacher. After all paragraphs of the second text had been read, in the same way has described above, the teacher said that the class was over.

\section{Evidence collection}

In this second case, 14 evidences were collected with 8 of them relative to the avatar, and 6 relative to the physical space. There are several units of analysis without any evidence in this case, namely "appearance", "gestures made", "sounds emitted", "eye gaze", and "facial demeanor" (Figure 17). For the other units of analysis, there were two pieces of evidence for "facial orientation", "body position," "avatar placement", "animated visual artifacts or interaction", and "not animated visual artifacts." The other units of analysis had one piece of evidence each. The collected pieces of evidences are compiled in Tables 7 and 8.

\section{Evidence analysis}

In this case, no evidences were found related to the first two propositions. Instead, the third proposition is supported by a chain that begins with the students sitting in the front row, while the responsible for the class (which behaved as a teacher, so we considered that he has indeed that role, and we refer to him as such in the analysis, and conversely, we refer to the other avatars as students). He stood in front of the students, and began the lesson. These units could change positions, i.e. both the attitude of the students can indicate that they want to attend the class, as well as the teacher's attitude may indicate that he wants to start it. Probably a bit of both are true, but at least one of them is. The fourth proposition is also supported by only a chain of evidence, which starts on the teleporter that was used to bring all participants to the room after indicated by the teacher and his example.

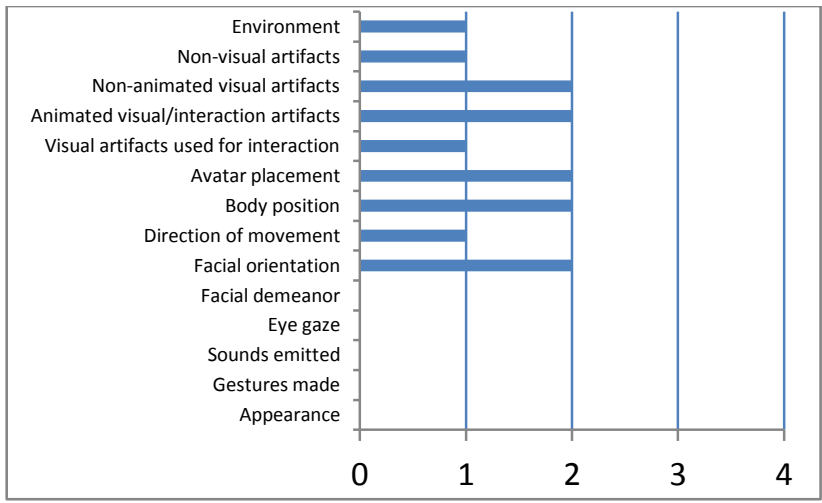

Figure 17. Distribution of evidences throughout the units of analysis (Case 2).

Table 7. Evidences related to the avatar (Case 2).

Facial orientation

The students sitting facing the panel across the room.

The teacher facing the students.

\section{Direction of movement}

The teacher moving towards a teleporter. 


\section{Body position}

The students sitting.

The teacher standing.

\section{Avatar placement}

The students sitting on the front row.

The teacher standing next to the stage.

\section{Visual artifacts used for interaction}

The voice icon over the avatar's heads.

Table 8. Evidences related to the physical space (Case 2).

\section{Animated visual artifacts (animated objects) or interaction (pose balls, teleport objects, etc.)}

Boxes (chairs) arranged as in a theatre.

Teleporter.

\section{Not animated visual artifacts}

Panels (schedule, payment, symbols)

Stage.

\section{Non-visual artifacts (eg. texts and scripts)}

Note cards.

\section{Visual environment}

Classroom.

The fifth proposition has three chains of evidence. The first is about the boxes that function as stools, with a disposition which is common in classrooms, helping students know where to sit. The second is about the panels that serve as references, since they are opposite to the chairs in the room, reinforcing the purpose of these. The third chain also reinforces this idea, since there is a stage opposite to the chairs, next to which the teacher stood. The sixth proposition has a chain related with note cards with the text that students should read. The teacher named the reader, and the other students could follow the reading on their note-card while listening. The seventh proposition is supported by the chain regarding the living room, previously referred to by the teacher. By that, the students became aware of the alleged use of the space. These chains are summarized in Table 9 and their distribution throughout the propositions is presented in Figure 18.
Table 9. Chains of evidences (Case 2).

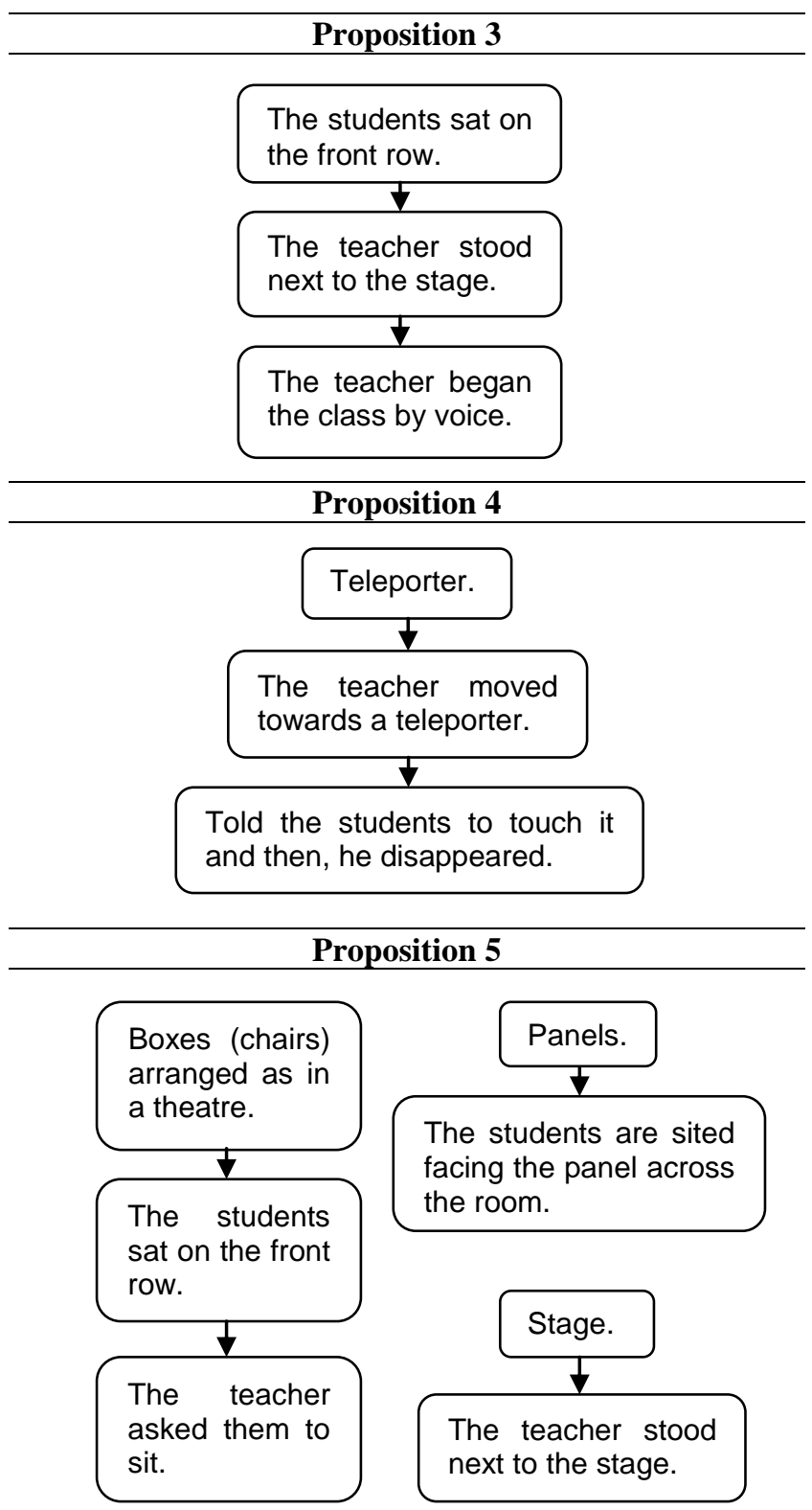

\section{Proposition 6}

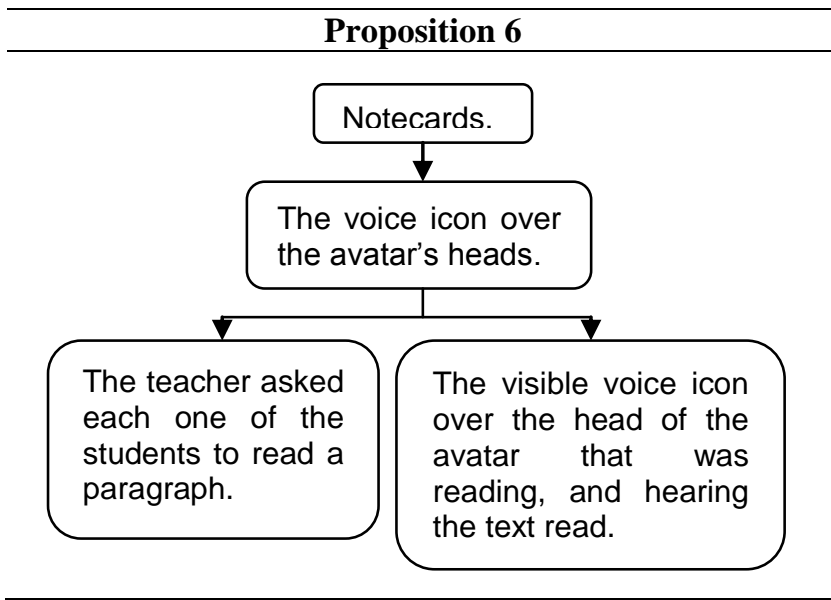



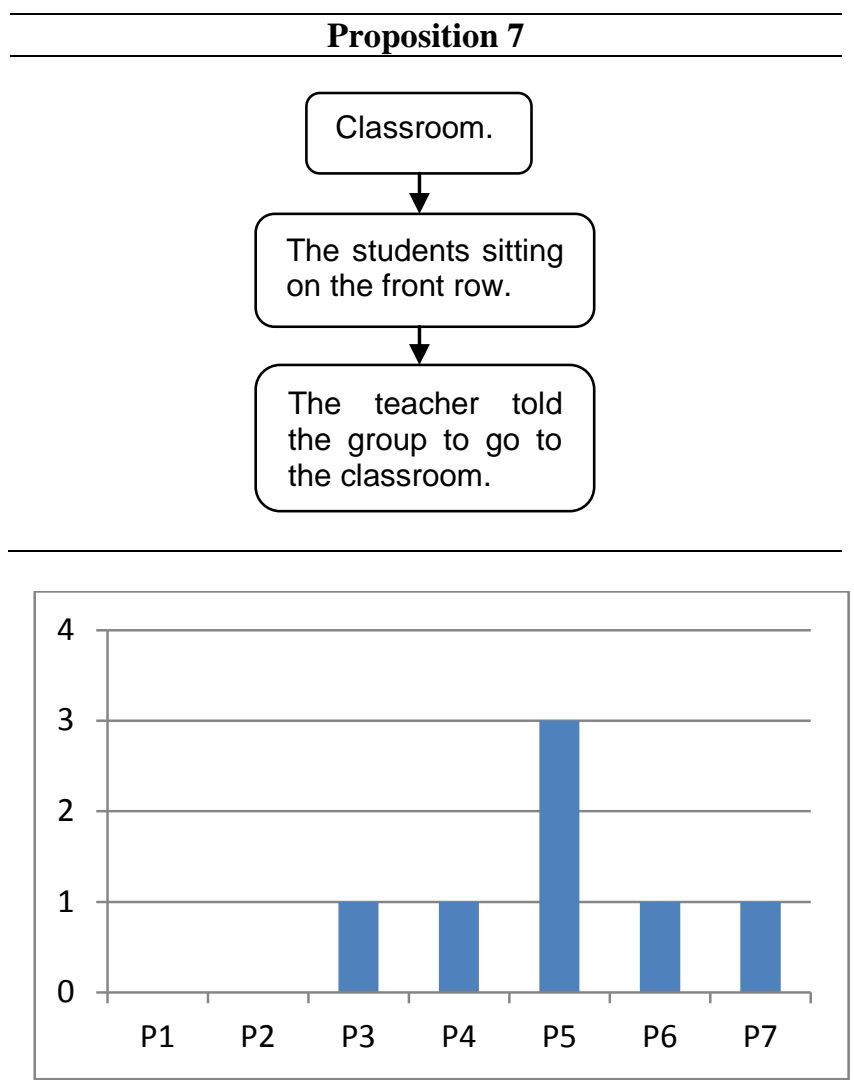

Figure 18. Distribution of the chains of evidences throughout the propositions (Case 2).

\section{Conclusion}

In this paper we presented a protocol for case study research of Presence and collaboration in virtual worlds, according to Yin's methodology. The propositions were defined and validated with a pilot case. The pilot case was held on Second Life, in a common scenario, and with a well known and easy to recreate subject: a class to teach how to build objects. After the description of the case itself, we analyzed it by extracting from the description examples of behaviours related to each unit of analysis. Then, chains of evidences were created by relating evidences based on the fact that each time a behaviour of an avatar or group of avatars, had as consequence, behaviours on other avatars. These cause/consequence relations where used to support the propositions. It was possible to find at least one chain of evidences for each proposition.

The protocol was then used in two cases, to obtain several chains of evidences supporting all but the first proposition. Based on the graphics presented on Figures 14 and 17, is possible to acknowledge that several units of analysis never occurred in these cases. Despite that fact, all but the first proposition were supported by at least one chain of evidence. We emphasize that these two cases were very similar cases. With different cases we are confident that all propositions may find support, as seen in the pilot case. Having in mind the need for more case studies to have a more solid base of validation of the propositions, by providing evidences for other units of analysis, it is nevertheless possible to draft answers to the research questions presented earlier. The first four propositions are related to the first question by referring to avatars' behaviours. Thus, the units of analysis related to the avatar will be used to answer the first question. Similarly, the other propositions are related to the physical space, so the units of analysis related to it will be used to answer the second question.

"Appearance", "Sounds emitted", and "Facial orientation" are the units of analysis that do not have any evidences in these two cases, but "Gestures made" does have. As do "Eye gaze", "Facial orientation", "Direction of movement", "Avatar placement", and "Visual artifacts used for interaction". So, it is possible to say that the use of an avatar influences collaboration in 3DVW by:

- gestures made by the avatar, influencing the perception by others of how the avatar's user wants to collaborate or how he/she wishes others to collaborate;

- avatar's eye gaze, facial orientation, direction of movement, or placement, which provide cues about what the user is paying attention to, or to what the user would like to direct others' attention towards;.

- artifacts with which the avatar interacts, providing cues about which objects are intended to be used by others in the collaboration process.

All units of analysis related to the virtual spatial environment have evidences in the two cases presented. It is therefore possible to state that the virtual spatial environment influences collaboration in $3 \mathrm{DVW}$ by:

- the animated visual artifacts, artifacts used for interaction, non-animated visual artifacts, and non-visual artifacts, through their arrangement, their specific features, or their purpose, which provide cues about their purpose for collaboration, helping define the team, contributing to group awareness, and perception of collaboration roles;

- the visual environment/virtual spatial environment, whose light, sounds/music, and visual effects can influence the attitudes of collaborators.

The evidence analysis of these cases relates evidences in chains that lead to the acknowledgment of a relation between an event caused by an avatar, and the reaction to it by other avatars, or their reaction to physical cues. By this, we can better understand the significance of the nonverbal communication used by the participants. Thus, these propositions and units of analysis can be used in 
future research for evidence collection and analysis on 3DVW when used for collaboration. Nevertheless, this process should be replicated with more case studies to provide multiple sources of chains, and so better support the propositions in order to build confidence on them.

\section{Acknowledgements.}

This work is financed by the FCT - Fundação para a Ciência e a Tecnologia (Portuguese Foundation for Science and Technology) within project UID/EEA/50014/2013.

\section{References}

[1] Pelet, J., Lecat, B., \& Papadopoulou, P. Enhancing learning and cooperation through digital virtual worlds. 2011 International Conference on Education, Research and Innovation (p. IPEDR vol.18). Singapore: IACSIT Press, 2001.

[2] Schaf, F., Paladini, S., \& Pereira, C. 3D AutoSysLab Prototype - A Social, Immersive and Mixed Reality Approach for Collaborative Learning Environments. In iJEP (Volume 2, Issue 2), 2012.

[3] Fominykh, M., Prasolova-Førland, E., \& Leong, P. Formal and Informal Collaborative Learning in 3D Virtual Campuses. Sixth International Conference on Collaboration Technologies. Sapporo, Japan, 2012.

[4] Owens, D., Davies, A., Murphy, J., Khazanchi, D., \& Zigurs, I. Real-World. IT Pro (pp. 34-41). IEEE, 2009.

[5] Hendaoui, A., \& Thompson, M. 3D Social Virtual Worlds - Research Issues and Challenges. Architectural Perspectives (pp. 88-92), 2008.

[6] Kristel M. De Nobrega, A.-F. R. Fostering Group Collaboration in Virtual Worlds. 2012 45th Hawaii International Conference on System Sciences - HICSS (pp. 983-992). IEEE, 2012.

[7] Campbell, M. Using 3D-virtual worlds to teach decision-making. Proceedings ascilite. Auckland, 2009.

[8] Panagopoulos, T., Jankovska, I., \& Straupe, I. Second Life 3D City Virtual Environment as an Urban Planning Tool for Community Engagement. In Recent Researches in Environmental Science and Landscaping. ISBN: 978-1-61804-090-9.

[9] Dreher, C., Reiners, T., Dreher, N., \& Dreher, H. 3D Virtual Worlds Enriching Innovation and Collaboration in Information Systems Research, Development, and Commercialisation. 3rd IEEE International Conference on Digital Ecosystems and Technologies (pp. 168-173). IEEE, 2009.

[10] Wordlswide, K. (n.d.). Retrieved at 2012, from http://www.kzero.co.uk

[11] Pinkwart, N., \& Oliver, H. Cooperative virtual worlds - A viable ecollaboration pathway or merely a gaming trend? Electronic Markets (Vol. 19, No. 4, pp. 233236), 2009.

[12] Yee, N. The Demographics, Motivations and Derived Experiences of Users of Massively-Multiuser Online Graphical Environments. PRESENCE: Teleoperators and Virtual, 2006.

[13] Benford, S., Greenhalgh, C., Rodden, T., \& Pycock, J. Collaborative Virtual Environments. Communications of the ACM , pp. 79-85, 2001.
[14] International Society for Presence Research. (n.d.). Retrieved at 2013, from International Society for Presence Research: http://ispr.info/

[15] Cruz, A., Fonseca, B., Paredes, H., Morgado, L., \& Martins, P. Can presence improve collaboration in 3D virtual worlds? SLACTIONS 2013: Research Conference on Virtual Worlds - Learning With Simulations (pp. Vol. 13; 47-55). Vila Real: Elsevier Procedia Technology, 2014.

[16] Romano, D., Brna, P., \& Self, J. Collaborative Decision-Making and Presence in Shared Dynamic Virtual Environments. Presence in Shared Virtual Evironments Workshop, 1998.

[17] Otto Otto, O., Roberts, D., \& Wolff, R. A Study of Influential Factors on Effective Closely-Coupled Collaboration based on Single User Perceptions. PRESENCE 2005 - 8th Annual International Workshop on Presence, 2005.

[18] Jordan Jordan, J., Mortensen, J., Oliveira, M., \& Slater, M. Collaboration in a Mediated Haptic Environment. Presence 2002: 5th Annual International Workshop on Presence. Porto, 2002.

[19] Romano, D., \& Brna, P. Collaboration, Presence and Performance in Virtual Learning Environments: Can Collaboration be Used to Measure Shared Presence? Presence 2002: 5th Annual International Workshop on Presence. Porto, 2002.

[20] Sadagic, A., Towles, H., Holden, L., Daniilidis, K., \& Zeleznik, B. Tele-immersion Portal: Towards an Ultimate Synthesis of Computer Graphics and Computer Vision Systems. PRESENCE 2001: The 4th Annual International Workshop on Presence, 2001.

[21] Kaltenbrunner, M., \& Huxor, A. Multiple Presence through Auditory Bots in Virtual Environments. Presence 2000: The Third International Workshop on Presence, 2000.

[22] Franceschi, K., \& Lee, R. Virtual Social Presence for Effective Collaborative E-Learning. PRESENCE 2008 - Proceedings of the 11th Annual International Workshop on Presence, 2008.

[23] Rae, J., Guimaraes, E., \& Steptoe, W. Simulation versus Reproduction for Avatar Eye-Gaze in Immersive Collaborative Virtual Environments. PRESENCE 2008 - Proceedings of the 11th Annual International Workshop on Presence, 2008.

[24] Gül, L. Affording Embodiment in Collaborative Virtual Environments: What is the Role of Presence in Collaborative Design? PRESENCE 2008 Proceedings of the 11th Annual International Workshop on Presence, 2008.

[25] Bente, G., Rüggenberg, S., Nicole, \& Krämer, C. Social Presence and Interpersonal Trust in AvatarBased, Collaborative Net-Communications. PRESENCE 2004-7th Annual International Workshop on Presence, 2004.

[26] Bente, G., \& Krämer, N. Virtual gestures: Analyzing social presence effects of computer-mediated and computer-generated nonverbal behaviour. Presence 2002: 5th Annual International Workshop on Presence. Porto, 2002.

[27] Gyorfi, J., Buhrke, E., Tarlton, M., Lopez, J., \& Valliath, G. VICC: Virtual Incident Command Center. PRESENCE 2005 - 8th Annual International Workshop on Presence, 2006.

[28] Otto, O., Roberts, D., \& Wolff, R. A Study of Influential Factors on Effective Closely-Coupled 
Collaboration based on Single User Perceptions. PRESENCE 2005 - 8th Annual International Workshop on Presence, 2005.

[29] Swinth, K., \& Blascovich, J. Perceiving and Responding to Others: Human-Human and HumanComputer Social Interaction in Collaborative Virtual Environments. Presence 2002: 5th Annual International Workshop on Presence. Porto, 2002.

[30] Cruz, A., Paredes, H., Fonseca, B., Martins, P., Morgado, L. Fitting Three Dimensional Virtual Worlds into CSCW. 2015 IEEE 19th International Conference on Computer Supported Cooperative Work in Design (CSCWD), Falerna Marina, Italy; $01 / 2015$

[31] Yin, R. K. Case study research: Design and methods. In Applied Social Research Series, Vol. 5., 4th edition. London: Sage, 2009.

[32] Crotty, M. (1998). The foundations of social research: Meaning and perspective in the research process. Sydney, Australia: Allen \& Unwin.

[33] Creswell, J. W. (2003). Research Design: Qualitative, Quantitative, and Mixed Methods Approaches. Thousand Oaks, London, \& New Delhi: SAGE Publications.

[34] Borrego, M., Douglas, E. P., \& Amelink, C. T. (2009). Quantitative, Qualitative, and Mixed Research Methods in Engineering Education. Journal of Engineering Education, 53-66.

[35] Olds, B. M., Moskal, B. M., \& Miller, R. L. (2005). Assessment in Engineering Education: Evolution, Approaches and Future Collaboration. Journal of Engineering Education, 13-25.

[36] Case, M. J., \& Light, G. (2011). Emerging Methodologies in Engineering Education Research. Journal of Engineering Education, 186-201.

[37] Doody, 0. N. (2013). Preparing and conducting interviews to collect data. Nurse Researcher, pp. 20, 5, 28-32.

[38] Boyce, C., \& Neale, P. (2006). CONDUCTING INDEPTH INTERVIEWS: A Guide for Designing and Conducting In-Depth Interviews for Evaluation Input. Pathfinder International.

[39] Leonard-Barton, D. A dual methodology for case studies: Synergistic use of a longitudinal single site with replicated multiple. Organization Science 1 (3), pp. 248-266, 1990.

[40] Gummesson, E. Qualitative methods in management research. Lund, Norway: Studentlitteratur, ChartwellBratt, 1998.

[41] Schramm, W. Notes on case studies for instructional media projects. Academy of EducationalDevelopment. Washington DC.

[42] Hartley, J. Case studies in organizational research. In C. C. Symon, Qualitative methods in organizational research: A practical guide (pp. 209-229). London: Sage, 1994.

[43] Sykes, W. Validity and reliability in qualitative market research: A review of the literature. Journal of the Market Research Society 32 (3) , 289-328, 1990.

[44] Brown, B., \& Bell, M. CSCW at play: ' There' as a collaborative virtual environment. CSCW 04. Chicago, USA: ACM, 2004. 